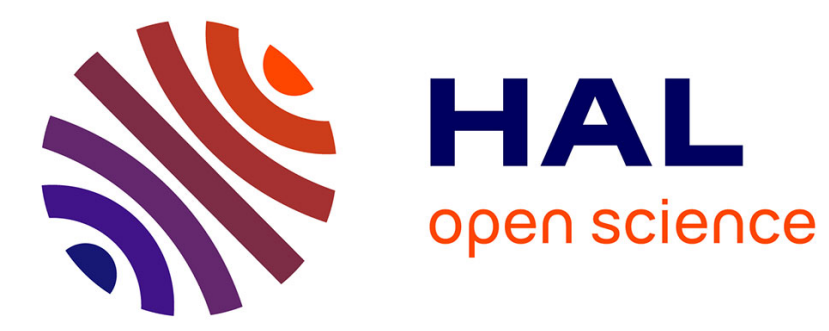

\title{
Nonlinear visco-elasticity of soft tissues under cyclic deformations
}

\author{
Martine Ben Amar
}

\section{To cite this version:}

Martine Ben Amar. Nonlinear visco-elasticity of soft tissues under cyclic deformations. International Journal of Non-Linear Mechanics, 2018, 106, pp.238-244. 10.1016/j.ijnonlinmec.2018.07.002 . hal01971729

\section{HAL Id: hal-01971729 \\ https://hal.sorbonne-universite.fr/hal-01971729}

Submitted on 7 Jan 2019

HAL is a multi-disciplinary open access archive for the deposit and dissemination of scientific research documents, whether they are published or not. The documents may come from teaching and research institutions in France or abroad, or from public or private research centers.
L'archive ouverte pluridisciplinaire HAL, est destinée au dépôt et à la diffusion de documents scientifiques de niveau recherche, publiés ou non, émanant des établissements d'enseignement et de recherche français ou étrangers, des laboratoires publics ou privés. 


\title{
Nonlinear visco-elasticity of soft tissues under cyclic deformations
}

\author{
Martine Ben Amar ${ }^{1}$ \\ Laboratoire de Physique Statistique, Département de Physique de l'ENS, PSL Research University, CNRS, Sorbonne Université, 75005 Paris
}

Sorbonne Université, Institut Universitaire de Cancérologie, Faculté de médecine, 75013 Paris, France

\begin{abstract}
Nowadays, new experimental set-ups allow to determine the strains which appear in biological tissues under small controlled solicitations as stretch (uniaxial and bi-axial) or shear. Automatically recorded by camera, these data exhibit hysteresis even for very slow and weak forcing. The aim of this work is to revisit the modeling of hyper-elasticity and visco-elasticity for tissues with a rather disordered structure. Despite the weakness of the imposed stretch values, strong nonlinearities are observed for these tissues that polymeric gels do not show under the same conditions. Various classical models have been tested on surgical mammary tissues and it turns out that the simplest Neo-Hookean model with its viscous counter-part explain perfectly the data. Viscosity and elasticity compete quite equivalently showing that, in the selected range of forcing values, visco-elasticity cannot be discarded.
\end{abstract}

Keywords: Finite elasticity and visco-elasticity; cyclic deformations, hysteresis, living fibrotic tissues

\section{Introduction}

As the description of fibrous tissues evolves and makes progress [1, 2], experimental set-ups have also been improved allowing the automatic determination of stress-strain results which can be compared to modelling of complex hyper-elastic tissues. Some theoretical models originate from a microscopic analysis of the structural properties of the sample under tension and the changes which may occur when a tensional state is applied. The case of elongation of tendons is an example 10 [3, 4]; the micro-structures have been analyzed in details, the link existing between the main components: collagen fibers, extra-cellular matrix and proteo-glycans, has been incorporated in the formalism with the aim to deduce a stress-strain compared to experimental data [5, 6]. Elaborated models can be established where the involved parameters rely on microscopic quantities as done for composite materials [7]. Hysteresis and plasticity behaviors can also be recovered. Such studies require a profound knowledge of the biological structures and of the evolving interactions between them under stress conditions.

20 However, they remain specific to the system under study and need to be validated by stress-strain experimental curves, when they are available.

Using different approaches, biophysics groups have elaborated a characterization of the material properties going from individual cells [8] to tissues. After investigated in detail isolated cell behavior during mitosis for example or migration of connected cells in an epithelium on a solid substrate [9], they show that

\footnotetext{
Email address: benamar@lps.ens.fr,DOI : https://doi.org/10.1016/j.ijnonlinmec.2018.07.002 (Martine Ben Amar) 1
}

collections of cells, interacting with each other and/or with the extra-cellular matrix, may exhibit emergent properties, which do not necessarily mirror the properties of their constitutive elements. An integrated approach is then necessary at the scale of the tissue leading to continuous visco-elastic modelling where fibers are treated using the formalism of nematic liquid crystal [10, 11]. Nonetheless, not all biological tissues exhibit a well defined organization. Let us mention the brain and fat tissues, [12, 13, 14], or fibrotic tissues [15] which play an important role in organogenesis but also in the development of pathologies: wound-healing [16, 17], capsules around implants [18], sarcoma (the generic word for cancer of tissues) and various tumors of the brain. Even if these tissues present an order at the microscopic scale, like bundles of fibers with an averaged orientation [1], phenomenological constitutive laws with a small number of parameters can be derived. Often, they intervene in the complex geometry of organs and a simplified constitutive law takes all its value to understand biological processes such as growth [19, 20] in embryo-genesis or in pathologies.

Parameters of constitutive laws may be experimentally deduced from compression/tension-shear tests under a sequence of multiple cycles [21]. These experiments exhibit hysteretic cycles which suggest that visco-elasticity plays an important role. However, if macroscopic constitutive laws exist for tissues like the Mooney-Rivlin [22], Ogden [23, 24], Gent [25], or the GOH [1] models, macroscopic viscoelasticity [26] do not offer the same diversity.

Indeed, a brief history of nonlinear dissipation modeling can be found in [27], going back to the beginning of the last century and a presentation of the so-called Landau model, commonly used in acoustics [28]. In the spirit of Landau, the theory of visco-elasticity rests on the definition of a dissipation energy 
60 rate, formulated in the same way as the elastic energy density, then introducing two new "Lamé" parameters for the viscoelastic part. But, as shown by Destrade and coworkers, to deduce correctly the viscous nominal stress or the viscous Cauchy stress is not so trivial. Fundamental properties such as objectivity (frame-indifference) or symmetry of the Cauchy stress are not always verified in the acoustic literature where the Landau's model is commonly accepted. Applying these symmetry arguments, they derive the correct viscous tensor for the Landau's model. Having in mind the fact that hyper-elasticity and 70 visco-elasticity must represent the same intrinsic tissue properties, being two sides of the same coin, it is proposed here a treatment to systematically derive the viscous tensor for an arbitrary representation of the hyper-elastic tissue properties. A variational method based on the extremum of the dissipation rate also called the Rayleighian [29, 30] in polymer physics is used. For each biomechanical constitutive law we show that we can construct easily its visco-elastic counter-part, and for each nominal or Cauchy stress, also its visco-elastic counterpart. As an example, a test on classical models is done for 80 fibrotic mammary tissues at very low stretch, less than 1.2. A cascade of hysteric loops are obtained when cycles of extensions/compression are applied. Strong nonlinearities appear that are analyzed with this formalism. The objective is to recover the stress- strain curves for the uniaxial stretch of a cuboid for low values of the stretch: $\lambda \sim 1$ as shown in Fig.(1).

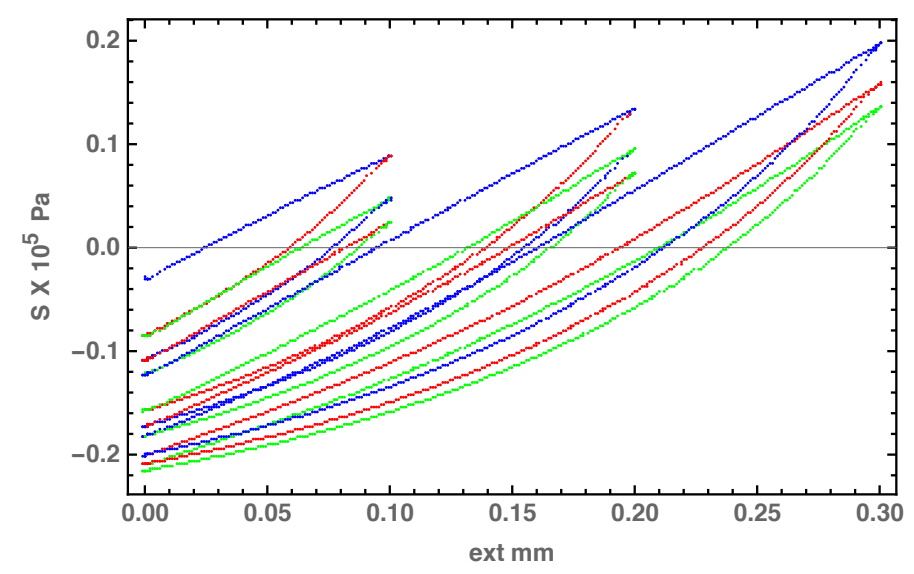

Figure 1: A typical set of measurements, force versus extension, concerning a surgical fibrotic tissue. Several cycles are made with the same maximal extension but with 3 different values for the final extension. There is a systematic shift towards lower values as the number of cycles increases.

\section{Constitutive laws for hyper-elastic tissues}

This section is just a reminder of 4 different models of nonlinear elasticity, which will serve as a basis for the derivation of 100 a nonlinear dissipation formalism. We focus on the Mooney-

90 Rivlin [22], Ogden [23, 24], Landau [31] and GOH [1, 2] models of hyper-elastic tissues. Their standard representation varies, sometimes introducing principal stretches or principal invariants of the Cauchy-Green tensors.

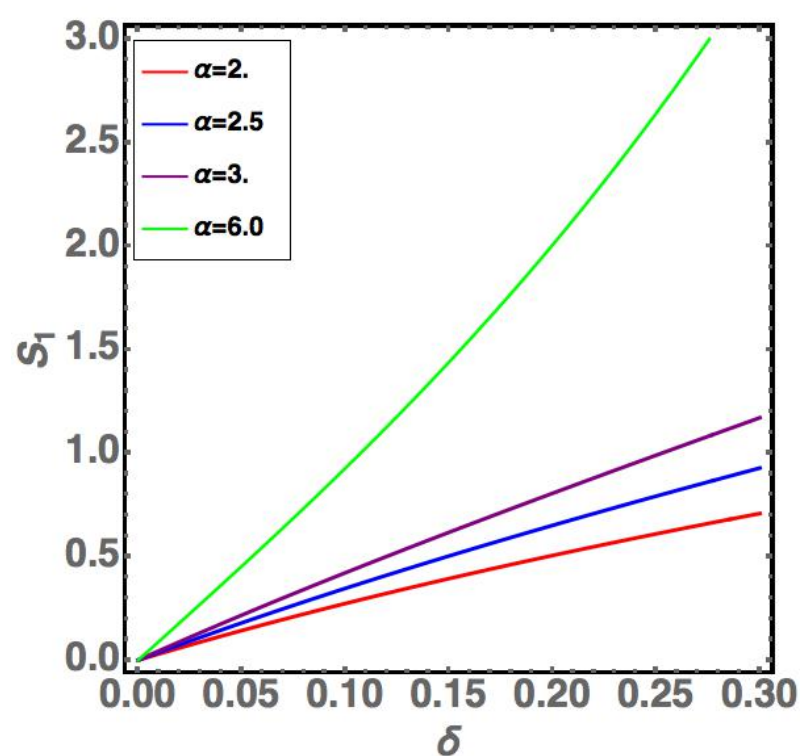

Figure 2: Nominal stress $S_{1}$ as a function of the vertical stretch in the Ogden model given by Eqs. 410 . Being a scaling parameter, $\mu_{p}$ is chosen to 1 . Notice the change of curvature in the stress answer for rather large exponents due to nonlinearities but in this example, the extension is ten times the values reached in Fig. (1)

\subsection{The Mooney-Rivlin model}

We begin with the Mooney-Rivlin model, which is restricted to two invariants $I_{1}$ and $I_{2}$, for incompressible materials since $I_{3}=1$.

$$
W_{M R}=\frac{\mu}{2}\left\{\left(I_{1}-1\right)+\rho\left(I_{2}-1\right)\right\}
$$

with $I_{1}=\operatorname{Tr}[\mathbf{C}]$ and $I_{2}=1 / 2\left(\operatorname{Tr}[\mathbf{C}]^{2}-\operatorname{Tr}\left[\mathbf{C}^{2}\right]\right)$, C representing the right Cauchy-Green strain tensor: $\mathbf{C}=\mathbf{F}^{\mathbf{T}} \mathbf{F}$ if $\mathbf{F}$ is the deformation gradient. $\operatorname{Tr}[\mathbf{A}]$ means the trace of the tensor $\mathbf{A}$. The shear modulus $\mu$ is positive, $\rho$ can be negative, but only to some extent. Keeping in mind the standard tensorial relationships (see [32],p 41-42).

$\frac{\partial \operatorname{Tr}[\mathbf{C}]}{\partial \mathbf{C}}=\mathbf{I} ; \frac{\partial \operatorname{Tr}\left[\mathbf{C}^{\alpha}\right]}{\partial \mathbf{C}}=\alpha \mathbf{C}^{\mathbf{T}(\alpha-\mathbf{1})}=\alpha \mathbf{C}^{\alpha-\mathbf{1}}$ and $\frac{\partial \operatorname{Det}[\mathbf{C}]}{\partial \mathbf{C}}=\mathbf{C}^{-\mathbf{1}}$

The Cauchy stress is then:

$$
\sigma_{e}=2 \mathbf{C} \frac{\partial W_{M R}}{\partial \mathbf{C}}-p=\mu\left(\left(1+3 \rho I_{1}\right) \mathbf{C}-\rho \mathbf{C}^{2}\right)-p \mathbf{I}
$$

where $p$ is the Lagrange parameter which ensures the incompressibility property.

\subsection{The Ogden model}

The Ogden model, introduced for rubber elasticity, turns out to be more and more employed in recent works on living tissues such as [12, 18, 21]. Its classical formulation is given in term of the principal stretches $\lambda_{i}$ for each axis of coordinate $X_{i}$ but different variants can be found in the literature:

$$
\begin{aligned}
W_{O g} & =\frac{\mu_{p}}{2 \alpha_{p}} \quad\left(\lambda_{1}^{2 \alpha_{p}}+\lambda_{2}^{2 \alpha_{p}}+\lambda_{3}^{2 \alpha_{p}}-3\right) \\
& +\frac{\mu_{p}}{2 \alpha_{p}} \quad \rho\left(\lambda_{1}^{-2 \alpha_{p}}+\lambda_{2}^{-2 \alpha_{p}}+\lambda_{3}^{-2 \alpha_{p}}-3\right)
\end{aligned}
$$


which can replaced by:

$$
W_{O g}=\frac{\mu_{p}}{2 \alpha_{p}}\left\{\operatorname{Tr}\left[\mathbf{C}^{\alpha_{\mathbf{p}}}\right]-3+\frac{\rho}{2}\left(\operatorname{Tr}\left[\mathbf{C}^{\alpha_{\mathbf{p}}}\right]^{2}-\operatorname{Tr}\left[\mathbf{C}^{2 \alpha_{\mathbf{p}}}\right]-6\right)\right\}
$$

when $\alpha_{p}$ is integer. Only the two-term Ogden model is presented but in principle a series expansion is possible varying $\alpha_{p}$ and $\mu_{p}$. According to Eq. (3), we can deduce the nominal stress:

$$
\sigma_{e}=\mu_{p}\left\{\left(\mathbf{C}^{\alpha_{\mathbf{p}}}+\rho\left(\mathbf{C}^{\alpha_{\mathbf{p}}} \operatorname{Tr}\left[\mathbf{C}^{\alpha_{\mathbf{p}}}\right]-\mathbf{C}^{2 \alpha_{\mathbf{p}}}\right)\right\}-p \mathbf{I}\right.
$$

Let us give the first component as:

$$
\sigma_{e, 1}=\mu_{p} \lambda_{1}^{2 \alpha_{p}}\left(1+\rho\left(\lambda_{2}^{2 \alpha_{p}}+\lambda_{3}^{2 \alpha_{p}}\right)\right)-p
$$

\subsection{The non-linear Landau model}

The nonlinear Landau model is just a nonlinear version of the Hookean model where the standard deformation tensor is replaced by the Green-Lagrange strain tensor, $\mathbf{E}=\frac{1}{2}\left(\mathbf{F}^{\mathbf{T}} \mathbf{F}-\mathbf{I}\right)=$ $\frac{1}{2}(\mathbf{C}-\mathbf{I})$. It seems to have been proposed by Brillouin [33] and turns out to be attributed to Landau, nowadays. For this model, the strain energy density is commonly given in term of the Green-Lagrange strain tensor, $\mathbf{E}=\frac{1}{2}\left(\mathbf{F}^{\mathbf{T}} \mathbf{F}-\mathbf{I}\right)=\frac{1}{2}(\mathbf{C}-\mathbf{I})$ as an expansion of third order. It is justified by the fact that it is directly related to the linear Hookean energy. Restricted to the first leading contributions, it reads:

$$
W_{L a}=\frac{\lambda_{0}}{2}(\operatorname{Tr}[\mathbf{E}])^{2}+\mu_{0} \operatorname{Tr}\left[\mathbf{E}^{2}\right]
$$

where $\lambda_{0}$ and $\mu_{0}$ are equivalent to the Lamé coefficients in linear elasticity (see [31]). This expansion is commonly called the Landau model in acoustic [28]. It is different from the MooneyRivlin expansion for hyper-elasticity since such expansion involves $I_{1}, I_{1}^{2}$ and $I_{2}$. Indeed after elementary algebra, it reads:

$$
W_{L a}=\frac{c_{1}}{4}\left(I_{1}-3\right)+\frac{c_{2}}{2}\left(I_{2}-3\right)+\frac{c_{3}}{8}\left(I_{1}^{2}-9\right)
$$

with $c_{1}=-\left(2 \mu_{0}+3 \lambda_{0}\right), c_{2}=-\mu_{0}$ and $c_{3}=\left(2 \mu_{0}+\lambda_{0}\right)$, while the Mooney-Rivlin model does not include $I_{1}^{2}$. In addition, incompressibility in linear elasticity means $\lambda_{0}$ infinite, indicating that this model may appear inadequate for living tissues. It is not difficult to deduce the stress tensor using Eq. (3):

$$
\sigma_{e}=\frac{\lambda_{0}}{2}\left(I_{1}-3\right) \mathbf{C}+\mu_{0}\left(\mathbf{C}^{2}-\mathbf{C}\right)-p \mathbf{I}
$$

These models treat all directions equivalently, they are isotropic so a priori do not involve fibers. The possible anisotropy of the fiber network which may play a role for fibrotic tissues is discarded. Let us consider now the GOH model.

\section{GOH model}

The Neo-Hookean model is recovered for $\rho=0$ in the Mooney-Rivlin definition (see Eq.(1)), for $\alpha_{p}=2$ in the oneterm Ogden model and for $c_{2}=c_{3}=0$ in the Landau model. The Mooney-Rivlen model corresponds to $\alpha_{p}=1$ for the Ogden model. The discussion for the GOH model is postponed to the next paragraph.

\section{Nonlinear viscoelasticity}

According to Destrade et al [27], some inconsistencies appear in the literature to establish the viscous dissipation tensor with the correct symmetry and the objectivity property although some issues can be found in [34]. Special attention is put on this question, focusing on the Landau's model. Based on fundamental principles of nonlinear mechanics, they derive a viscous stress tensor as:

$$
\mathbf{S}_{L a, v d}=\tilde{\lambda}_{0}\left(\operatorname{Tr}(\dot{\mathbf{E}}) \mathbf{F}+2 \mu_{0} \mathbf{F} \dot{\mathbf{E}}\right.
$$

where $\tilde{\lambda}_{0}$ and $\tilde{\mu}_{0}$ are chosen by analogy with Eq. 8 but having different values. Here, we suggest another way to recover this result, perhaps more simple and which can be generalized to arbitrary hyper-elasticity modeling with its extension to viscoelasticity. Considering first the Landau model,we define an energy dissipation rate per unit time, in the same spirit as Eq.80:

$$
\left.R_{L a}=\frac{\tilde{\lambda}_{0}}{2}(\operatorname{Tr}[\dot{\mathbf{E}}])^{2}+\tilde{\mu}_{0} \operatorname{Tr}\left[\dot{\mathbf{E}}^{2}\right]\right)
$$

The full energy variation rate also called the Rayleighian [30, 29] of the system is then:

$$
\begin{aligned}
\mathcal{R} & =\int_{V} d V\left(\frac{d W_{\mathbf{E}}}{d t}-p \dot{J}+R_{\dot{\mathbf{E}}}\right) \\
= & \int_{V} d V\left\{\left(\frac{\partial W_{\mathbf{E}}}{\partial \mathbf{E}}-p \frac{\partial J}{\partial \mathbf{E}}\right) \dot{\mathbf{E}}+R_{\dot{\mathbf{E}}}\right\}
\end{aligned}
$$

Its extremum, with respect to an arbitrary elastic strain rate requires to consider :

$$
\delta \mathcal{R}=\int_{V} d V\left(\frac{\partial W_{\mathbf{E}}}{\partial \mathbf{E}}-p \frac{\partial J}{\partial \mathbf{E}}+\frac{\partial R_{\dot{\mathbf{E}}}}{\partial \dot{\mathbf{E}}}\right): \delta \dot{\mathbf{E}}
$$

The first two terms in Eq. 15 is nothing else that $\mathbf{F}^{-\mathbf{1}} \mathbf{S}_{e}$ where $\mathbf{S}_{e}$ is the nominal stress. By analogy, we can define the viscous stress by imposing that the last term in Eq. 15 is equal to $\mathbf{F}^{-1} \mathbf{S}_{v d}$ so that the total stress is $\mathbf{S}=\mathbf{S}_{e}+\mathbf{S}_{v d}$. The integrand becomes $\mathbf{F}^{-\mathbf{1}} \mathbf{S}: \delta \dot{\mathbf{E}}=\operatorname{Tr}\left[\mathbf{F}^{-\mathbf{1}} \mathbf{S} \delta \dot{\mathbf{E}}\right]$ since the tensor $\mathbf{F}^{-\mathbf{1}} \mathbf{S}$ is selfadjoint. We will show now that, by imposing the stationarity of the Rayleighian with respect to any variation of the local velocity, we recover the classical law of the nonlinear elasticity, exactly in the same way as the stationary energy principle. Using the cyclic property of the Trace, it reads:

$$
\begin{gathered}
\delta \mathcal{R}=\int_{V} d V \operatorname{Tr}\left[\mathbf{F}^{-1} \mathbf{S}\left(\delta \dot{\mathbf{F}}^{\mathbf{T}} \mathbf{F}+\mathbf{F}^{\mathbf{T}} \delta \dot{\mathbf{F}}\right)\right. \\
=\int_{V} d V \operatorname{Tr}\left[\mathbf{S}^{\mathbf{T}} \delta \dot{\mathbf{F}}\right]=\int_{V} d V \mathbf{S}: \operatorname{Grad} \frac{\partial \vec{r}}{\partial t}
\end{gathered}
$$

By standard integration by part for the last integral, the classical equilibrium equation is recovered Div $\mathbf{S}=0$ but for a dissipative system and the border condition: $F(t) \cdot \vec{N}=\mathcal{A S} \cdot \vec{N}$ , where $\mathcal{A}$ is the transverse area of the sample, perpendicular to the normal $\vec{N}$. Defining $\Sigma=F(t) / \mathcal{A}$, the Kelvin-Voigt model is recovered for nonlinear hyper-elastic tissues. In addition, in the case of the Landau's model, the Destrade et al. result is found without difficulty (Eq.(11)) since:

$$
\mathbf{F}^{-1} \mathbf{S}_{v d}=\frac{\partial R_{\dot{\mathbf{E}}}}{\partial \dot{\mathbf{E}}}=\tilde{\lambda}_{0} \operatorname{Tr}[\dot{\mathbf{E}}] \mathbf{I}+2 \tilde{\mu}_{0} \dot{\mathbf{E}}
$$


With this variational method, based on the extremum of the dissipation rate, no assumption is required. The viscous stress $\mathbf{S}_{v d}$ simply results from the first principles of mechanics of dissipative systems with a proper choice of dissipation energy. Concerning hyper-elastic model given in term the Cauchy-Green tensor $\mathbf{C}$ a similar dissipation energy rate $R_{\dot{\mathbf{C}}}$ is expected to be given in term of $\dot{\mathbf{C}}$, then the variational method again applies with this formulation and we get:

$$
\sigma_{v d}=2 \mathbf{C} \frac{\partial R_{\dot{\mathbf{C}}}}{\partial \dot{\mathbf{C}}} \quad \text { or } \quad \mathbf{S}_{v d}=2 \mathbf{F} \frac{\partial R_{\dot{\mathbf{C}}}}{\partial \dot{\mathbf{C}}}
$$

Illustration of this formulation is given in the next section with application to the cuboid stretching.

\section{Viscous dissipative stress tensor and stress distribution for a cuboid under uniaxial tension}

Coming back to the Ogden model for the hyper-elastic energy, Eq. (4), the dissipation rate can be formulated as follows:

$$
R_{O g}=\frac{\tilde{\mu}_{p}}{2 \alpha_{q}}\left\{\operatorname{Tr}\left[\dot{\mathbf{C}}^{\alpha_{\mathbf{q}}}\right]+\frac{\tilde{\rho}}{2}\left(\operatorname{Tr}\left[\dot{\mathbf{C}}^{\alpha_{\mathbf{q}}}\right]^{2}-\operatorname{Tr}\left[\dot{\mathbf{C}}^{2 \alpha_{\mathbf{q}}}\right]\right)\right\}
$$

The nominal stresses, deduced from the energy density $W_{O g}$ (Eq. (5) reads:

$$
\mathbf{S}_{O g}=\mu_{p} \mathbf{F} \mathbf{C}^{\alpha_{\mathbf{p}}-\mathbf{1}}\left(1+\rho\left(\operatorname{Tr}\left[\mathbf{C}^{\alpha_{p}}\right]-\mathbf{C}^{\alpha_{\mathbf{p}}}\right)-p \mathbf{F}^{-\mathbf{T}}\right.
$$

and the viscous stress tensor becomes :

$$
\mathbf{S}_{O g, v d}=\tilde{\mu}_{p} \mathbf{F} \dot{\mathbf{C}}^{\alpha_{\mathbf{q}}-\mathbf{1}}\left\{1+\tilde{\rho}\left(\operatorname{Tr}\left[\dot{\mathbf{C}}^{\alpha_{\mathbf{q}}}\right]-\dot{\mathbf{C}}^{\alpha_{\mathbf{q}}}\right)\right\}
$$

We consider the simplest case of a cuboid in the vertical direction (called one), with a ramp linear in time $\dot{\lambda}_{1}$ (being the velocity of elongation divided by the initial length of the sample). We assume incompressibility and both boundaries 2 and 3 are free of stress. The stretch $\lambda_{2}$ and $\lambda_{3}$ are equal and the incompressibility imposes the following relations:

$$
\lambda_{2}=\lambda_{3}=\frac{1}{\sqrt{\lambda_{1}}} \quad \text { and } \quad \dot{\lambda}_{2}=\dot{\lambda}_{3}=-\frac{1}{2} \lambda_{2} \frac{\dot{\lambda}_{1}}{\lambda_{1}}=-\frac{1}{2} \frac{\dot{\lambda}_{1}}{\lambda_{1}^{3 / 2}}
$$

Let us define each component of the nominal stress by separating the elastic and viscous part such: $\mathbf{S}_{O g, i}=S_{i}-p / \lambda_{i}$, it reads:

$$
\begin{gathered}
S_{1}=\mu_{p} \lambda_{1}^{2 \alpha_{p}-1}\left(1+2 \rho \lambda_{1}^{-\alpha_{p}}\right) \\
S_{2}=\mu_{p} \lambda_{1}^{-\alpha_{p}+1 / 2}\left(1+\rho\left(\lambda_{1}^{2 \alpha_{p}}+\lambda_{1}^{-\alpha_{p}}\right)\right)
\end{gathered}
$$

For the viscous stress tensor, it reads:

$$
\begin{gathered}
S_{1, v d}=\tilde{\mu}_{p} 2^{\alpha_{q}-1} \lambda_{1}^{\alpha_{q}} \dot{\lambda}_{1}^{\alpha_{q}-1}\left\{1+2 \tilde{\rho}\left(\frac{-\dot{\lambda}_{1}}{\lambda_{1}^{2}}\right)^{\alpha_{q}}\right\} \\
S_{2, v d}=\tilde{\mu}_{p} \lambda_{1}^{-1 / 2}\left(\frac{-\dot{\lambda}_{1}}{\lambda_{1}^{2}}\right)^{\alpha_{q}-1}\left\{1+\tilde{\rho}\left(2 \lambda_{1} \dot{\lambda}_{1}\right)^{\alpha_{q}}+\left(\frac{-\dot{\lambda}_{1}}{\lambda_{1}^{2}}\right)^{\alpha_{q}}\right\}
\end{gathered}
$$

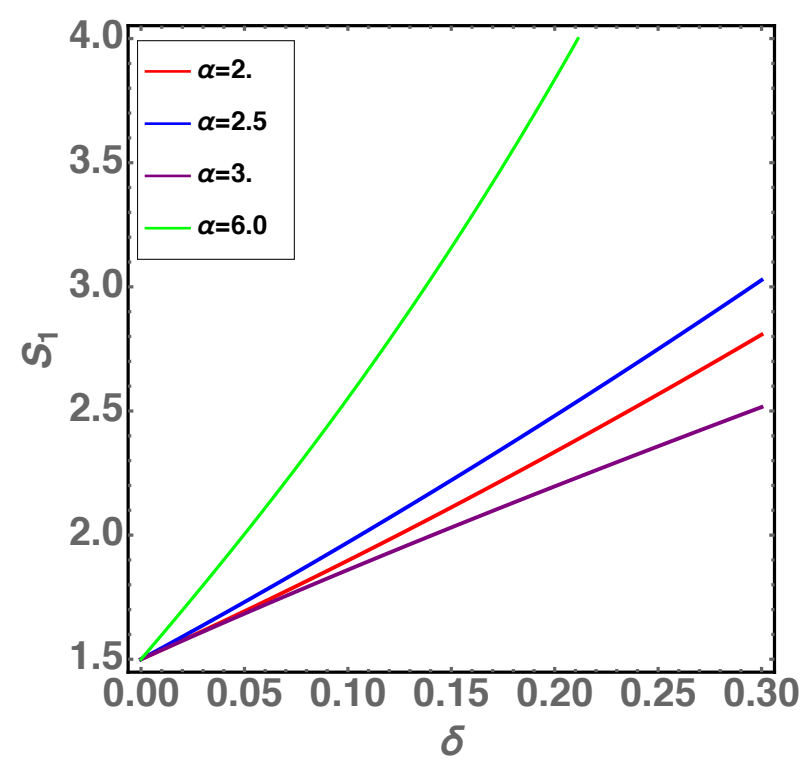

Figure 3: Nominal stress $S_{1}$ taking into account both the elastic and viscoelastic stresses according to Eq. (27 30). The linear ramp is $\dot{\lambda}_{1}=1$, the viscous coefficients are: $\tilde{\lambda}_{0}=\tilde{\mu}_{0}=0.5$.

Cancellation of the total stress in the second and third components, free at the boundaries, gives the value of $p$ and we obtain finally the stress component of interest as:

$$
S_{O g, 1}=S_{1}+S_{1, v d}-\frac{1}{\lambda_{1}^{3 / 2}}\left(S_{2}+S_{2, v d}\right)
$$

So the total stress is decomposed into an elastic and a viscouselastic part which may be the analogous of a non-linear version of the Kelvin-Voigt model. Let us give the result for the Mooney-Rivlin model [22]:

$$
S_{1}=\mu_{p}\left(\left(\lambda_{1}-\frac{1}{\lambda_{1}^{2}}\right)+\rho\left(1-\frac{1}{\lambda_{1}^{3}}\right)\right)
$$

while for the viscous stress, we take $\alpha_{q}=2$ since $\alpha_{q}=1$ has no physical meaning. This emphasizes that, for the dissipation, the degree of nonlinearities is not the same as for the elasticity. In this case, we derive

$$
S_{v 1}=\tilde{\mu}_{p} \dot{\lambda}_{1}\left(\left(\lambda_{1}^{-4}+2 \lambda_{1}^{2}+\tilde{\rho} \dot{\lambda}_{1}^{2} \lambda_{1}^{-8}\left(1+8 \lambda_{1}^{6}\right)\right)\right.
$$

For the Landau dissipation model we get:

$$
S_{v 1}=\frac{\dot{\lambda}_{1}}{\lambda_{1}^{4}}\left(\tilde{\lambda}_{0}\left(\lambda_{1}^{3}-1\right)^{2}+\tilde{\mu}_{0}\left(1+2 \lambda_{1}^{6}\right)\right)
$$

All these models are isotropic which may be sufficient for low stretch values. However, it may be important to consider also fibrous anisotropic tissues which play an important role in pathologies but also in embryogenesis [35] as demonstrated recently by laser nano-indentation [36] .

\subsection{The GOH model}

Nonlinearity and visco-elasticity are expected to be stronger for fibrotic tissues. It is why we also present the results for 
the GOH model [1] and its possible visco-elastic counterpart. This model induces strong nonlinearities and has been proved to exhibit the correct behavior in a previous work on the same kind of fibrotic tissues [18]. For multiple families of fibers, it allows to introduce an averaged orientation and a possible dispersion for each family:

$$
W_{G O H}=\frac{\mu}{2}\left\{(\operatorname{Tr}[\mathbf{C}]-3)+\Sigma_{j} \frac{k_{j, 1}}{2 k_{j, 2}}\left(e^{k_{j, 2}[\mathbf{C}: \mathbf{H}-1]^{2}}-1\right)\right\}
$$

where $k_{i, j}$ 's are positive dimensionless stiffness parameters. $k_{j, 1}$ is related to the stiffness of the fibers in the small strain regime while $k_{j, 2}$ is related to large strain stiffening behavior of the fibers [27]. Considering no specific orientation for the fibers, the model simplifies becoming:

$$
W_{G O H}=\frac{\mu}{2}\left\{\operatorname{Tr}[\mathbf{C}]-3+\frac{k_{1}}{2 k_{2}}\left(e^{\left.k_{2}(\operatorname{Tr}[\mathbf{C}]-3)-1\right)}\right)\right\}
$$

We can imagine that the visco-elasticity dissipation rate obeys the same rules giving:

$$
R_{G O H, v d}=\frac{\tilde{\mu}}{8}\left\{\operatorname{Tr}\left[\dot{\mathbf{C}}^{2}\right]+\frac{\tilde{k}_{1}}{\tilde{k}_{2}}\left(\left(e^{\tilde{k}_{2}\left(\operatorname{Tr}\left[C^{2}\right]-\dot{C}^{2}\right)}\right)\right\}\right.
$$

It is then easy to calculate the viscous stress, for example in the direction 1

$$
S_{G O H, v d}=\tilde{\mu} \lambda_{1}^{2} \dot{\lambda}_{1}\left(1+\tilde{k}_{1} e^{4 \tilde{k}_{2}\left(\left(\dot{\lambda}_{1} \lambda_{1}\right)^{2}+\left(\dot{\lambda}_{2} \lambda_{2}\right)^{2}\right)}\right)
$$

with equivalent expression for the other components.

Since our apparatus imposes ramps of extension, linear in time, the force to apply is given by the initial horizontal surface of the sample times the value of the nominal stress $S_{1}$ which is plotted in Fig. 23 for different values of the elastic model and different values for the ramp velocities. Independently of the model used for the dissipative contribution, there is a jump in tension as soon as the loading ramp is applied. This jump at zero static stretch is not observed in our data, hidden by the calibration. If this model may represent conveniently the nonlinear counterpart of the Voigt viscoelasticity model, for the Maxwell model it does not seem so obvious. So we will accept without any more demonstration the linear Maxwell model [37] joined to the nonlinear Voigt model. One important question concerns the existence of pre-stretch or pre-stress for these tissues. The pre-stress accumulated by the pathology seems to have disappeared but pre-stretch is possible. In case of pre-stretch one has to revisit our determination of $S_{1}$ by a modification of $\lambda_{1}$ by $\lambda_{1} / \lambda_{p}, \lambda_{2}$ by $\lambda_{2} / \sqrt{\lambda_{p}}$ and $\lambda_{3}$ by $\lambda_{3} / \sqrt{\lambda_{p}}$.

\section{The nonlinear Maxwell-Kelvin-Voigt Model}

A simple glance to our Fig.(1) indicates that it will be impossible to recover the data data with only the Kelvin-Voigt approach whatever the model. The experiment is realized with a constant ramp so $\dot{\lambda}_{1}$ is a constant. This has been checked by analyzing the data automatically recorded by a camera, the applied load is effectively applied with a constant ramp and the absolute value is the same in the ascending and descending parts.

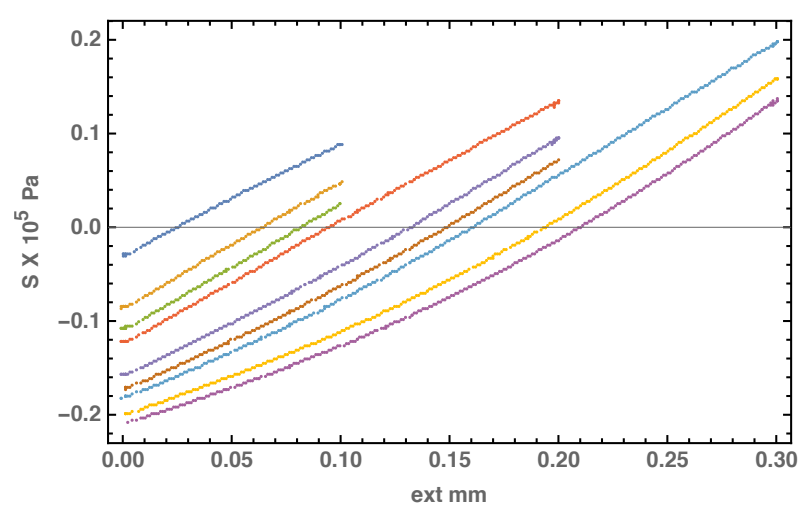

Figure 4: The 9 stress/extension curves obtained experimentally for one specific sample of mammary fibrotic tissue. The measurement corresponds to the nominal stress. For clarity, we only show the results for ascending ramp obtained with a velocity of $0.01 \mathrm{~mm} / \mathrm{s}$. The extension is given in $\mathrm{mm}$ and the length of the sample is $1 \mathrm{~cm}$.

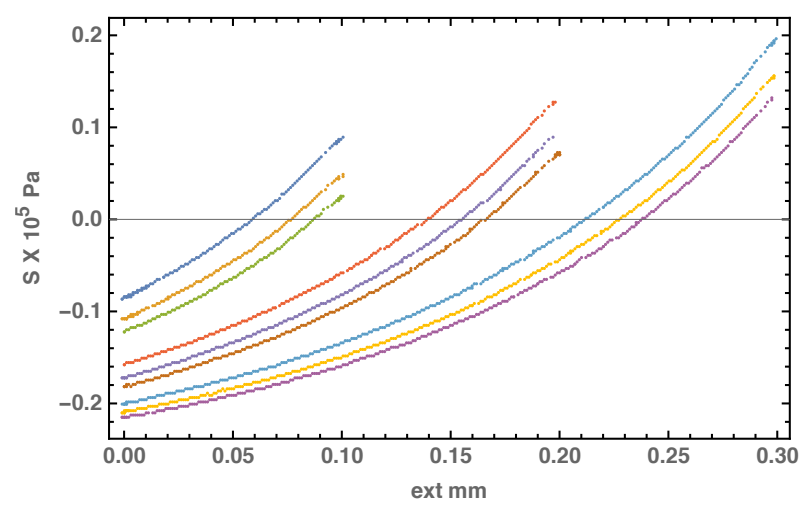

Figure 5: The 9 stress/extension curves obtained experimentally for one specific sample of mammary fibrotic tissue. The measurement corresponds to the nominal stress. For clarity, we only show the results for a descending ramp obtained with a velocity of $0.01 \mathrm{~mm} / \mathrm{s}$. The extension is given in $\mathrm{mm}$ and the length of the sample is $1 \mathrm{~cm}$.

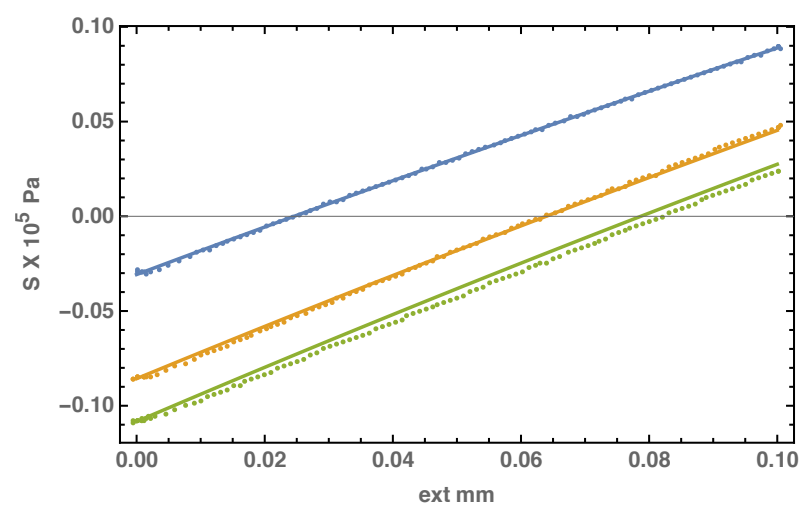

Figure 6: Superposition of the nominal stress obtained experimentally and theoretically in the Neo-Hookean model, see Eqs. 28 29]. For clarity, we only show the results for the first and last ascending ramps obtained with a velocity of 0.01 $\mathrm{mm} / \mathrm{s}$ and the first spindle of curves, with a maximal extension of $0.1 \mathrm{~mm}$. The extension is given in $\mathrm{mm}$ and the length of the sample is $1 \mathrm{~cm}$, corresponding to a stretch of $\lambda_{1}=1.01$ 


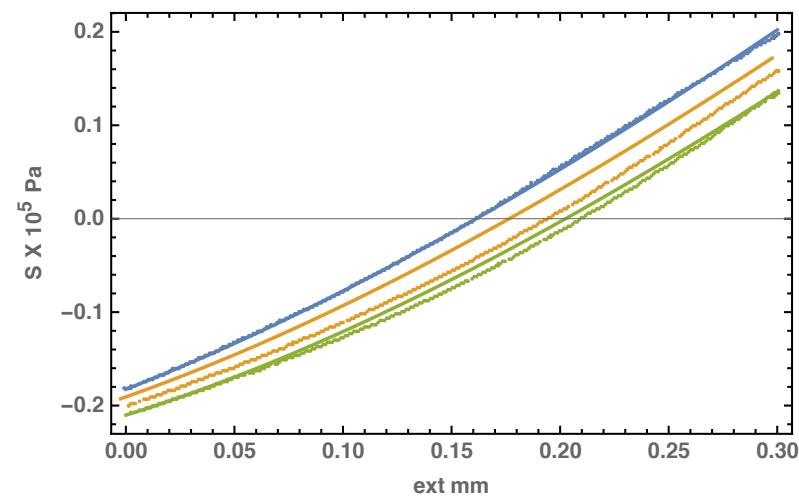

Figure 7: Superposition of the nominal stress obtained experimentally and theoretically in the Neo-Hookean model, see Eqs. 28[29]. For clarity, we only show the results for the first and last ascending ramps obtained with a velocity of $0.01 \mathrm{~mm} / \mathrm{s}$ and for the last spindle of curves, with a maximal extension of $0.3 \mathrm{~mm}$, corresponding to a stretch of $\lambda_{1}=1.03$. The extension is given in $\mathrm{mm}$ and the length of the sample is $1 \mathrm{~cm}$.

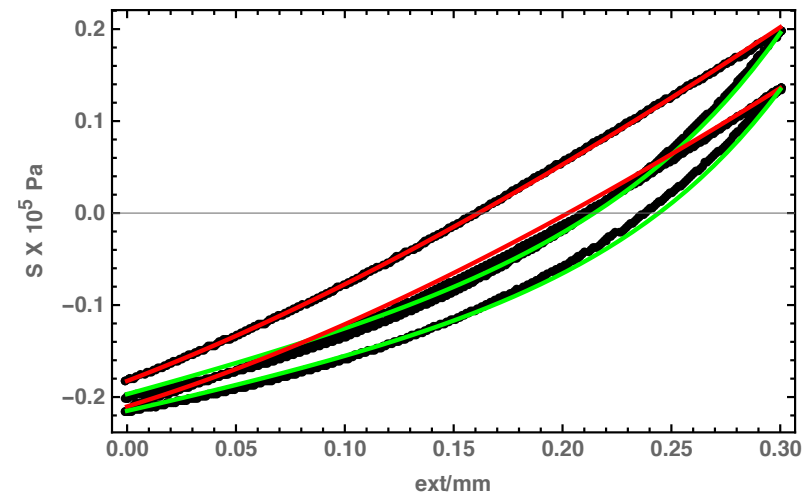

Figure 8: The stress/extension curves obtained in the first and third rounds of the last set (see Fig. 1. Experimental data are represented by black lines.180 Analytical results are given by red curves for the first round trip, in green for the last one. The extension is given in $\mathrm{mm}$ and the length of the sample is $1 \mathrm{~cm}$. Exact values of the parameters can be found in Section 7
As a consequence, the viscous tensor contribution will consist in adding or subtracting a constant since the length extension remains small. So there is no hope that the models presented here may reproduce the data. In addition, there is a difficulty to extract the true value at time $t=0$ due to calibration of the apparatus. Whatever the value found at $\lambda=1$, it is obvious that we do not have the degree of freedom necessary to make a cycle. This is true when $\lambda_{1}$ increases but also when it decreases. The Maxwell model which introduces a viscous dissipation damping put in series with our Voigt nonlinear approach, may help to recover the cycles observed in Fig.(1)

$$
\eta \frac{d S}{d t}+S=S_{e 1}+S_{v 1}
$$

Since the left-hand side is directly deduced from linear elasticity, the choice of the Cauchy stress may be preferred although the force acting on the sample is directly proportional to the nominal stress. In [37] and references included, the choice is made of the nominal stress and this is the choice we finally adopt in the last section. The ramp being imposed, fixing $\dot{\lambda}_{1}$, this equation which as one degree of freedom allows to fix one value for the stress $S$ for the ascending ramp and for the decreasing ramp, then a cycle can be recovered giving hope to recover the data. Only a good fitting with a limited number of parameters can confirm the strategy. Notice that if we can estimate the parameters which intervene in the elastic and viscoelastic tensors, it is not the case for $\eta$. In particular we do not know if $\eta$ depends on the whole apparatus or on the tissue mechanical properties. $\eta$ has the dimension of a time. Let us now analyze the data shown in Fig.(1).

\section{Data versus Modelling}

In this section, preliminary results on cyclic deformations are presented for small extension of fibrotic and surgical tissues. All the spectra: uniaxial stress/extension realized at very small velocities $0.01 \mathrm{~mm} / \mathrm{s}$ exhibit the same appearance as in Fig(1) both for fibrotic and artificial surgical tissues. Our samples con70 sist in surgical fibrotic mammary tissues [18] which vary from one patient to another, depending on the grade of the pathology and the human variability. An extensive study will be the subject of future work. What is known is that these tissues contain a very disorganized collagenous structure. However, the sample treated here presents generic features which is not shared by elastomers for example, with the same dimensions. The details on the apparatus and experimental procedure can be found in [38]. The main difficulty of such tensional test are drainage effects consisting of exchange of water between the tissue and the bath which will spoil the measurements. So the ramp velocity is chosen very small to avoid these exchanges difficult to evaluate quantitatively. As shown in Fig.(1), nonlinearities of the stress/strain answer are very strong, even for a very low stretch value, less than 1.01. This is an amazing result but it has been observed on 4 samples. Indeed, if it strongly suggests dissipation, standard models as shown in Fig.(1) show more easily an increase of the stress in the negative ramp compared to the positive ramp. One may think of the so-called Mullins effect which 
has been introduced in the biomechanical literature to explain been on internal degree of freedom which varies with the applied stretch. An illustration for rubber-like materials has been given by Tommasi et al. ([39, 40]). These authors consider the microscopic structure of polymeric gels which can breakdown under stretching. This obviously modifies the elastic energy of the samples which contains less fibers due to the breaking of the chains. In [18], such a scenario is also investigated with eventually a reorientation of the fiber ${ }_{240}$ network in the direction of the stretching. However all these changes have little chance to occur for the rather low stretch 200 value, shown in Fig.(1) and for these models, the stress always cancel for zero stretch. Although, there is an uncertainty on the real zero in our experiment, these models cannot explain the systematic shift towards low stress values. Only dissipation can explain the gap observed at low vanishing stretching since all models give a value proportional to $\dot{\lambda}_{1}$. Unfortunately, the calibration of the zero appears to be uncertain and so we use the value at zero stretch as a parameter to include in the fitting process. The final results are shown in Figs. 5 4/67/8) allowing the following conclusions:

- At extremely stretch values, hysteretic cycles on fibrotic tissues are explained by a competition between the elastic energy and the dissipation.

- In this range of forcing values, the Neo-Hookean model, possibly improved by the Mooney-Rivlin, gives the most stable results between different cycles and different extensions (see the agreement between data and the NeoHookean models in Fig. (6) for ascending ramps, in Fig. (7) ) for descending ramps and for two cycles of the last set of curves,Fig. (8).

- The best models for the dissipation energy rates is given by Eqs. 28 29, so the dissipation based on the Neo-Hookean formulation. In particular, the Landau model, according to Eq. 30 (with $\tilde{\lambda}_{0} \neq 0$ ) gives relatively unstable results.

- A pre-stretch is necessary, larger than than one during a descending ramp, after an ascending ramp, smaller than one after a descending ramp. Although remaining very close to one, the quality of the fitting depends tremendously on its value.

- The coefficient $\eta$ of Eq. (35) is different for ascending/descending ramps but remains stable during the nine ${ }^{270}$ examples shown in fig 8 .

Finally, here are the results for the sample we have selected, beginning by the intrisic values of the material: the numerical parameters $\mu_{p}$ (Eq.28) and $\tilde{\mu}_{p}$ (Eq. 29) that we can estimate are:

$$
\mu_{p}=10^{5} \text { Pas and } \tilde{\mu}_{p}=0.1710^{8} \text { Pas.s }
$$

while for the viscous parameter according to Eq. (35), we get two different values, one for the ascending part, the other for ${ }^{280}$ the descending part as for both pre-stretch values:

$$
\eta_{A}=0.44 s ; \eta_{D}=0.08 s ; \lambda_{p, A}=0.955 \text { and } \lambda_{p, D}=1.006
$$

So, in this example, elasticity and visco-elasticity are in competition but nonlinearities for the stresses (right-hand side of Eq. 35 can be discarded. The stress/strain answer is essentially sensitive to the $\eta$ coefficient and the Maxwell viscosity. The pre-strain is probably due to a weak effect of orientation/ disorientation when the sample is stretched or compressed. However, it is a parameter which dramatically influences the agreement between the model and the experiment. Here we give an averaged value since it very slightly changes from one set of curves from another.

\section{Conclusion}

This approach is based on traditional models of biomechanics. Other modelings can be found and complexity can be added via the introduction of internal variables, maybe useful at larger stretch for fibrotic tissues. The theory of nematic elastomers have pointed out that the fibers play a crucial role in the sample response to unidirectional extension. It is complicated by the fact that, for incompressible materials, the extension in one direction induces automatically compression in one or two other directions with a different answer according to fiber orientations. In addition, if the fibers have a given preferred direction in average, this one may change during loading, becoming an internal variable. It is highly appreciated if measurements give values which are typical of the material and not of the measure itself, but in this simple set-up, measurements affect the internal structure even at rather low stretch value. A more systematic study of these tissues (either fibrotic or artificially reconstructed for plastic surgery), at larger extension values, will be the subject of future work.

\section{Acknowledgments}

MBA would like to thank the Isaac Newton Institute for Mathematical Sciences, Cambridge, for support and hospitality during the programme "Growth, Form and Self-Organization" where work on this paper was undertaken. MBA acknowledges the partial support from the Simons Foundation, the EPSRC grant no EP/K032208/1 and from ANR under the contract MecaTiss (ANR-17-CE30-0007). MBA thanks Khawla Sleiman and Fréderic Lechenault for their participation to the experiment and Dr Michael Atlan for providing surgical tissues.

[1] T.R. Gasser, R.W. Ogden and G. Hopzafel, Hyperelastic modelling of arterial layers with distributed collagen fibre orientation J.R.Soc. Interface 3 (2006) 15-35.

[2] R.W. Ogden, Biomechanical Modelling at the molecular, cellular and tissue levels CISM, Courses and Lectures (2009) 508.

[3] P. Ciarletta and M. Ben Amar A finite dissipative theory of temporary interfibrillar bridges in the extra-cellular matrix of ligaments and tendons, Proc. Roy. Soc. Interface 6 (2009)909-924.

[4] T Shearer, WJ Parnell, B Lynch, J.M. Allain, Sequential straightening and loading viscoelasticity: A model of the time-dependent behaviour of tendon fascicles incorporating fibril recruitment, preprint

[5] D.M. Pioletti and L.R. Rakotomanana, On the independence of time and strain effects in the stress relaxation of ligaments and tendons. . Biomech 33 (2000) 17291732. 
[6] A.N. Natali, P.G. Pavan, E.L. Carniel, M.E. Lucisano, and G. Taglialavoro, Anisotropic elasto-damage constitutive model for the biomechanical analysis of tendons. Med. Eng. Phys. 27 (2005) 209214. (doi:10.1016/j.medengphy. 2004.10.011)

[7] A.H. Muliana,J. Sik Kim,A concurrent micromechanical model for predicting nonlinear viscoelastic responses of composites reinforced with solid spherical particles.Int. J. of Sol. and Struct. 44(21)(2007),68916913

[8] L., LeGoff, H. Rouault, and T. Lecuit , A Global Pattern of Mechanical Stress Polarizes Cell Divisions and Cell Shape in the Growing Drosophila Wing Disc. Development (2013) 140(19) (2013) 4051-9.

[9] C. A.M. La Porta, A. Ghilardi, M. Pasini, L. Laurson, M. J. Alava, S. Zapperi, M. Ben Amar, Osmotic stress affects functional properties of human melanoma cell lines. E.P.J.Plus, 130 (2015) 64, 1-15.

[10] K. Kruse, J. F. Joanny, F. Jlicher, J. Prost, and K. Sekimoto. Asters, Vortices, and Rotating Spirals in Active Gels of Polar Filaments. Phys. Rev. Lett. 92, (2004) 078101104

[11] M. C. Marchetti, J. F. Joanny, S. Ramaswamy, T. B. Liverpool, J. Prost, Madan Rao, and R. Aditi Simha Rev. Mod. Phys. 85 (2013) 11431189.

[12] L.A. Mihai, L. Chin, J.A. Janmey and A. Goriely, A comparison of hyperelastic constitutive models applicable to brain and fat tissues.J. R. Soc. Interface 12 (2015) 0150486.

[13] L.A. Mihai, S. Budday, G.A. Holzapfel, E. Kuhl E., and A. Goriely, A family of hyperelastic models for human brain tissue. To appear Journal 380 of the Mechanics andPhysics of Solids doi:10.1016/j.jmps.2017.05.015

[14] Y., Jiang Y., G. Li, L.X. Qian, S. Liang S., M. Destrade and Y. Cao Measuring the linear and nonlinear elastic properties of brain tissue with shear waves and inverse analysis Biomech Model Mechanobiol 14 (2015) 11191128.

[15] M. Ben Amar and C. Bianca,Towards a unified approach in the modeling of fibrosis: A review with research perspectives, Physics of Life Reviews, Phys Life Rev. Jul;17, (2016) 61-85. doi: 10.1016/j.plrev.2016.03.005.

[16] M. Wu and M. Ben Amar Growth and remodelling for profound circular wounds in skin Biomech Model Mechanobiol. 14(2): (2015) 357370.

[17] L.G. Bowden, H.M. Byrne, P.K. Maini, D.E. Moulton, A morphoelastic model for dermal wound closure Biomech Model Mechanobiol. 15(3) (2016) 663-681.

[18] M. Ben Amar, M. Wu, M., Trejo M. and Atlan M. Morpho-elasticity of inflammatory fibrosis: the case of capsular contracture Journ. Roy. Soc. Interface 12 (2015) 20150343

[19] M. Ben Amar and F. Jia Anisotropic growth shapes intestinal tissues during embryo-genesis Proc. Nat. Acad. Sciences (PNAS) 110 (26) (2013) 10525-10530

[20] V. Balbi and P. Ciarletta Morpho-elasticity of intestinal vill.J R Soc Interface 13;10(82) (2013) 20130109. doi: 10.1098/rsif.2013.0109.

[21] S. Budday, G. Sommer, C. Birkl , C. Langkammer,J., Haybaeck , J. Kohnert, M. Bauer, F. Paulsen, P. Steinmann, E. Kuh, and G.A. Holzapfel, Mechanical characterization of human brain tissue Acta Biomaterialia $\mathbf{4 8}$ (2017) 1-550. doi: htp://dx.doi.org/10.1016/j.actbio.2016.10.036

[22] M. Mooney, A theory of large elastic deformation. J. Appl. Phys.11 (1940) 582592.

[23] R.W. Ogden Large deformation isotropic elasticity on the correlation of theory and experiment for incompressible rubberlike solids Proc. Roy. Soc. A,A326 (1972) 565-584.

[24] R.W. Ogden Non-linear elastic deformations Dover Publications and Ellis Horwood (1984)

[25] A.N. Gent A new constitutive relation for rubber. Rubber Chemistry Technol. 69 (1996) 5961.

[26] A. Winemann, Nonlinear viscoelastic solids-a review Math.Mech.Solids 14 (2009) 300-366

[27] M. Destrade, G. Saccomandi G. and M. Vianello,Proper formulation of viscous dissipation for nonlinear waves in solids The J Acoust Soc Am. 133 (2013)1255-1259.

[28] Ye W,Bel-Brunon A., Catheline S., CombescureA. And Rochette M. (2017) Simulation of non-linear transient elastography: finite element model for the propagation of shear waves in homogeneous soft tissues, preprint

[29] L.D. Landau and E.M. Lifshitz Statistical physics (Volume 5 of A Course of Theoretical Physics ) Pergamon Press (1970).

[30] M. Doi Onsager's variational principle in soft matter J. Phys. Cond Matt. 23 (2011) 284118.
[31] L.D Landau and E. M. Lifshitz, Theory of Elasticity ( Volume 7 of A Course of Theoretical Physics ) Pergamon Press (1970).

[32] G.A. Holzapfel Nonlinear Solid Mechanics a continuum approach for engineering Wiley (2000)

[33] L. Brillouin Sur les tensions de radiation (On tensions of radiation) Ann. Phys. 4 (1925) 528-86.

[34] R. De Pascalis, I.D. Abrahams and W.J.Parnell On nonlinear viscoelastic deformations: a reappraisal of Fung's quasi-linear viscoelastic model.Proc.R.Soc.A470 (2014) (2166):20140058.

[35] R. Clément, B. Dehapiot,C. Collinet,T. Lecuit, P. Lenne, Viscoelastic Dissipation Stabilizes Cell Shape Changes during Tissue Morphogenesis,Current Biology27 2017 , 31323142.

[36] T.T.K Vuong-Brender, M. Ben Amar,J. Pontabry and M. Labouesse,The interplay of stiffness and force anisotropies drive embryo elongation eLife 2017;6:e23866. DOI: 10.7554/eLife.23866

[37] T. Belytschko, W. Kam Liu, B. Moran, K.I. Elkhodary, Nonlinear Finite Elements for Continua and Structures, Wiley (2014).

[38] The apparatus consists in an INSTRON machine which only operates uniaxial testings but is equipped for experiments in a water bathconstant imposed temperature. The measured force is of order the Newton, the ramp velocity is $10^{-2} \mathrm{~mm} / \mathrm{s}$ and approximatively 100 data points are automatically recorded for the first ascending ramp, 200 for the second set of curves, 300 for the last one, giving a final number of points of order 36000.

[39] D. De Tommasi, G. Puglisi and G. Saccomandi, Localized versus diffuse damage in amorphous materials phys.Rev.Lett. 100 (8 (2008) 085502

[40] D. De Tommasi, G. Puglisi and G. Saccomandi, A micromechanics-based model for the Mullins effect Journal of Rheology50 (4) (2006) 495-512. 